\title{
Pseudogap in the chain states of $\mathrm{YBa}_{2} \mathrm{Cu}_{3} \mathrm{O}_{6.6}$
}

\author{
V. B. Zabolotnyy, ${ }^{1}$ A. A. Kordyuk, ${ }^{1}$ D. Evtushinsky, ${ }^{1}$ V. N. Strocov, ${ }^{2}$ L. Patthey, ${ }^{2}$ T. Schmitt, ${ }^{2}$ D. Haug, ${ }^{3}$ C. T. Lin, ${ }^{3}$ \\ V. Hinkov, ${ }^{4,3}$ B. Keimer, ${ }^{3}$ B. Büchner, ${ }^{1}$ and S. V. Borisenko ${ }^{1}$ \\ ${ }^{1}$ Institute for Solid State Research, IFW-Dresden, P. O. Box 270116, D-01171 Dresden, Germany \\ ${ }^{2}$ Paul Scherrer Institut, Swiss Light Source, CH-5232 Villigen PSI, Switzerland \\ ${ }^{3}$ Max-Planck-Institut für Festkörperforschung, Heisenbergstraße 1, D-70569 Stuttgart, Germany \\ ${ }^{4}$ Quantum Matter Institute, University of British Columbia, Vancouver, B.C. V6T 1Z1, Canada
}

(Received 24 October 2011; revised manuscript received 22 December 2011; published 8 February 2012)

\begin{abstract}
As established by scanning tunneling microscopy (STM), cleaved surfaces of the high-temperature superconductor $\mathrm{YBa}_{2} \mathrm{Cu}_{3} \mathrm{O}_{7-\delta}$ develop charge-density wave (CDW) modulations in the one-dimensional (1D) $\mathrm{CuO}$ chains. At the same time, no signatures of the $\mathrm{CDW}$ have been reported in the spectral function of the chain band previously studied by photoemission. We use soft x-ray angle-resolved photoemission spectroscopy to detect a chain-derived surface band that had not been detected in previous work. The $2 k_{\mathrm{F}}$ for the new surface band is found to be $0.55 \AA^{-1}$, which matches the wave vector of the CDW observed in direct space by STM. This reveals the relevance of the Fermi-surface nesting for the formation of CDWs in the $\mathrm{CuO}$ chains in $\mathrm{YBa}_{2} \mathrm{Cu}_{3} \mathrm{O}_{7-\delta}$. In agreement with the short-range nature of the CDW order the newly detected surface band exhibits a pseudogap whose energy scale also corresponds to that observed by STM.
\end{abstract}

DOI: 10.1103/PhysRevB.85.064507

PACS number(s): 71.45.Lr, 74.72.Kf, 79.60.Jv

\section{INTRODUCTION}

The $\mathrm{CuO}$ chain structure in $\mathrm{YBa}_{2} \mathrm{Cu}_{3} \mathrm{O}_{7-\delta}(\mathrm{Y}-123)$ provides a physical realization of a quasi-one-dimensional electronic system with nonvanishing coupling to the $\mathrm{CuO}_{2}$ bilayers. ${ }^{1,2}$ One of the reasons why one-dimensional (1D) electronic systems remain in the focus of solid-state research is that even weak interactions transform the quasiparticles of Fermiliquid theory into collective excitations of density wave type $^{3-5}$ In particular, the peculiar shape structure of the chain Fermi surface (FS) makes this electronic system prone to formation of charge-density wave via the Peierls instability. ${ }^{6}$ Indeed, charge-density modulations along the $\mathrm{Cu}-\mathrm{O}$ chains have been extensively studied probing cleaved surfaces of Y-123 in direct space with scanning tunneling microscopy. ${ }^{7-15}$ It has been shown that $\mathrm{Y}-123$ crystals cleave between the $\mathrm{CuO}$ chains and the $\mathrm{BaO}$ layer, resulting in surface patches terminated either by disrupted $\mathrm{CuO}$ chains or $\mathrm{BaO}$ layer. Therefore the chains turn out to be the nearest to the surface building block ${ }^{7,9,10,15}$ contributing to the spectral weight at the Fermi level (FL). ${ }^{1,2}$ Both the earliest ${ }^{9}$ and the most recent studies $^{14}$ present a consistent picture of a charge-density wave (CDW), appearing as corrugations of the electronic density with a short correlation range of about $40 \AA$ and a period between 9 and $14 \AA$, depending on the sample stoichiometry.

In the case of 2D systems, the effect of CDW on the electronic states in the reciprocal space has been examined in great detail with modern angle-resolved photoemission spectroscopy, which provides both momentum and energy resolution when measuring the one-particle spectral function. Occurrences of the pseudogap for an incommensurate (or short-range-ordered) state, which finally develops into a true CDW band gap below $T_{\mathrm{CDW}}$, are well documented. ${ }^{16-19} \mathrm{In}$ the case of 1D systems other than Y-123, modifications to the spectral function with the onset of the CDW state have been detected as well. ${ }^{20-22}$ The emergence of a pseudogaplike state is also expected in theory and can be understood as a result of a fluctuating Peierls order parameter. ${ }^{23-28}$
At the same time, despite the abundant evidence for charge modulations in direct space provided by scanning tunneling microscopy (STM), Y-123 chains seem to exhibit neither a pseudogap nor the Tomonaga-Luttinger behavior observed in the related $\mathrm{PrBa}_{2} \mathrm{Cu}_{4} \mathrm{O}_{8} \cdot{ }^{29,30}$ Momentum resolved spectra measured from as-cleaved Y-123 surfaces ${ }^{31-33}$ as well as those measured on the in situ doped ones ${ }^{34}$ manifest neither folding nor notable suppression of the spectral weight at the Fermi level. That is, the spectral features that could be regarded as signatures of the CDW state are absent in spectra of Y-123. Therefore it remains unclear why the two complementary methods [STM and angle-resolved photoemission spectroscopy (ARPES)] deliver such conflicting results.

\section{METHODS}

To address this problem we have investigated high quality Y-123 crystals using modern soft $\mathrm{x}$-ray (SX)-ARPES. the photoemission data for this study were collected at the recently built high-resolution soft $\mathrm{x}$-ray beamline ADRESS at the Swiss Light Source. ${ }^{35}$ This beamline delivers photon flux up to $10^{13} \mathrm{ph} / \mathrm{s}$ in an energy window of $0.01 \%$ of photon energy. Such a high flux allows one to break through the problem of dramatic reduction of the valence-band cross section at high photon energies. the samples were mounted on a lowtemperature goniometric manipulator (CARVING) with three angular degrees of freedom and cleaved in situ in ultrahigh vacuum with base pressure better than $5 \times 10^{-11} \mathrm{mBar}$ at $T=10 \mathrm{~K}$, the same temperature at which all the spectra were acquired. The energy resolution depends on the kinetic energy and will be stated in the figure captions; details of the SX-ARPES station will be published elsewhere. ${ }^{36}$ The experiments were performed using high quality single crystals of $\mathrm{YBa}_{2} \mathrm{Cu}_{3} \mathrm{O}_{6.6}\left(T_{\mathrm{c}} \approx 61 \mathrm{~K}\right)$ from the same batch as in the recent INS study ${ }^{37}$ and $\mathrm{YBa}_{2} \mathrm{Cu}_{3} \mathrm{O}_{6.4}\left(T_{\mathrm{c}} \approx 33 \mathrm{~K}\right)$. The crystals were synthesized by the solution-growth technique, annealed to the desired oxygen doping, and detwinned by applying uniaxial mechanical stress at elevated temperature. ${ }^{37}$ 
As a result of the "surface polar catastrophe",34,38 the electronic structure of the Y-123 surface layer is known to be different from that of the bulk. ${ }^{39}$ Generally, there are two major approaches that have been used in photoemission practice to highlight bulk states against the surface ones. Surface aging is the first option, which basically relies on the destruction of surface sates. ${ }^{40,41}$ The other approach employs the variation of photon excitation energy and polarization, which affects the inelastic escape depth of exited electrons ${ }^{42-44}$ and the ratio of photoemission matrix elements for bulk and surface states. ${ }^{33,45}$ Also the rapid variation in the elastic-scattering rate with the kinetic energy of the excited photoelectrons may play a decisive role. ${ }^{46,47}$

\section{RESULTS AND DISCUSSION}

We start out the discussion of experimental data with an illustration of the first option. Figure 1 contains a comparison
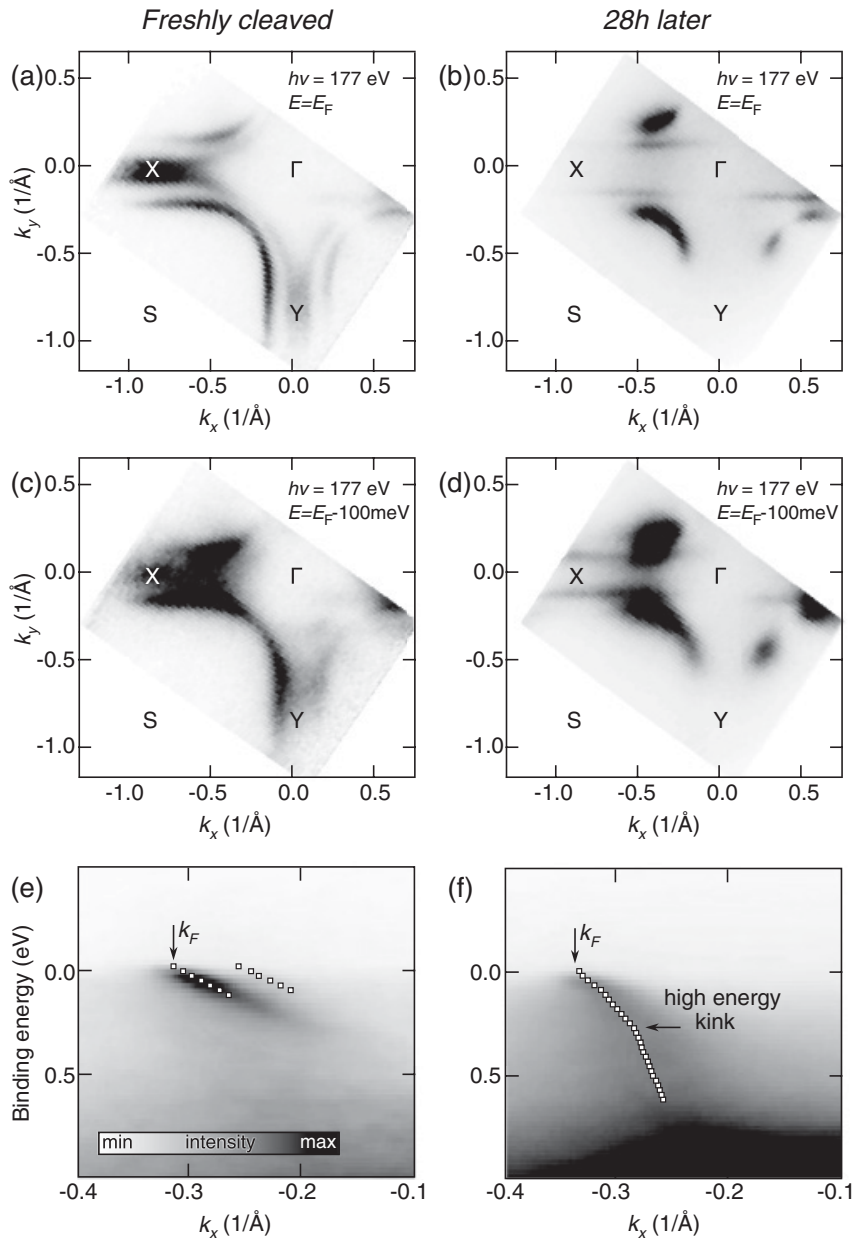

FIG. 1. Sample aging for $\mathrm{YBa}_{2} \mathrm{Cu}_{3} \mathrm{O}_{6.6}$. (a), (b) FS maps measured directly after cleavage and $28 \mathrm{~h}$ later. (c), (d) Corresponding fresh and aged intensity distributions for constant energy cuts at $E=$ $E_{\mathrm{F}}-100 \mathrm{meV}$. (e), (f) Energy-momentum intensity distributions along the $\Gamma-S$ direction. The white squares are the result of MDC fits. Dark corresponds to high photoemission intensity. Spectra measured at $T=10 \mathrm{~K}$ with $38-\mathrm{meV}$ energy resolution using linearly $s$-polarized light (i.e., no out-of-plane component of the polarization vector). of the Fermi surfaces measured from the freshly cleaved sample to the same measurement done $28 \mathrm{~h}$ after the cleavage. The freshly cleaved surface results in a typical picture of bilayer split FS contours corresponding to the $\mathrm{CuO}_{2}$ plane states (rounded double squares centered at the $S$ point) and the quasi-one-dimensional chain band (faint features running parallel to the $k_{x}$ axis). After surface degradation there are some notable changes.

First, the overdoped $\mathrm{CuO}_{2}$ plane bands are replaced with the "Fermi arc" features similar to those observed in Refs. 48 and 49. The appearance of the Fermi arcs is also accompanied by a $k_{\mathrm{F}}$ shift [Figs. 1(e)-1(f)]. For the bonding band, it can be estimated from the fits of momentum distribution curves (MDC), and amounts to about $\delta k_{\mathrm{F}} \approx 0.04 \AA$ along the $\Gamma-S$ direction. Assuming the same shrinking over the whole Fermi surface and an average FS radius $k_{\mathrm{F}} \approx 0.55 \AA$, one may estimate the decrease in hole doping as $\delta p \approx 4 \pi k_{\mathrm{F}} \delta k_{\mathrm{F}} / S_{\mathrm{BZ}} \approx$ 0.1 , which brings us from the typically overdoped regime with $p \approx 0.3$ (Refs. 31, 32, 40, and 50) under the superconducting dome with $p \approx 0.2$. Note also the so-called "waterfalls" and a high-energy kink at about $250 \mathrm{meV}$, which have recently generated an avalanche of publications. ${ }^{51-54}$ These issues are beyond the scope of the current study, and we do not further elaborate on them here.

Second, the relative intensity of the 1D chain band as compared to the 2D bands is substantially increased, while the distance between the chain Fermi crossings, $2 k_{\mathrm{F}}$, remains practically the same. This suggests that the observed chain band is most likely a "bulk" feature, in a sense that it originates from the chain structure protected by at least one $\mathrm{CuO}_{2}$ bilayer, and not from the cleaved chains. Since the upper neighboring $\mathrm{CuO}_{2}$ bilayers turn out to be overdoped, the electronic structure of these chains still differs from LDA band-structure calculation, ${ }^{2}$ so in the following we will refer to these chains as subsurface ones, in order to contrast this structure with the chains through which the cleavage takes place and the true bulk chains.

This observation is consistent with the interpretation given in Ref. 33, which was based on the pattern of circular dichroism. This work has shown that the chain signal observed in ARPES experiment mainly originates from the nearest to the surface undisturbed chains protected by the overdoped $\mathrm{CuO}_{2}$ bilayer, so that the surface aging is expected to enhance the photoemission from these chain states as compared to other surface features. Though there are intensity variations along the chain band, it is noteworthy that the band exhibits neither pseudogap nor folding phenomena. As can be seen in Figs. 1(a)-1(d), there are no indications for a CDW state for the freshly cleaved and aged surfaces.

Now we turn to the discussion of the second option. The so-called inelastic mean free path (IMFP) $\lambda$ has been a convenient measure for surface sensitivity of the $\mathrm{x}$-ray photoelectron spectroscopy when dealing with thin overlayer films. It has been experimentally demonstrated that attenuation of excited photoelectrons, passing through a deposited thin film, strongly depends on the kinetic energy, ${ }^{42-44}$ with the highest absorbtion observed for the energies in the range 30-100 eV. Therefore it is often believed that shifting to extremely low or high excitation energies $(\lesssim 10 \mathrm{eV} / \gtrsim 1 \mathrm{keV})$ should increase the "bulk sensitivity," even when one's aim is 

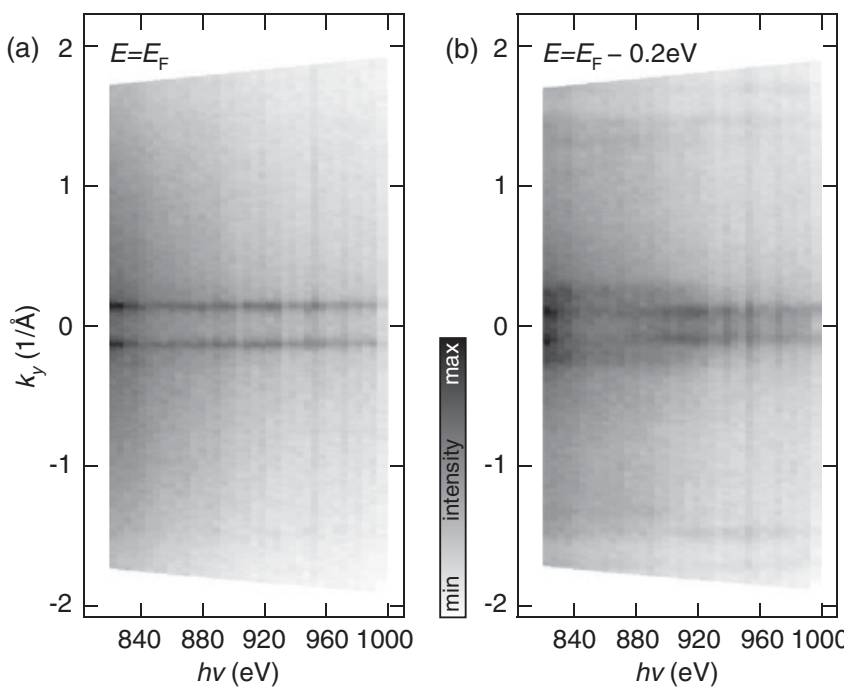

FIG. 2. Excitation energy dependence for the cut passing through the $\Gamma$ point parallel to the $k_{y}$ axis. The left panel shows the intensity integrated around the Fermi level, $E=E_{F}$. The right image contains intensity at $200 \mathrm{meV}$ below the Fermi level, demonstrating another set of chain features for $h v \lesssim 920 \mathrm{eV}$. Spectra were measured from $\mathrm{YBa}_{2} \mathrm{Cu}_{3} \mathrm{O}_{6.6}$ sample using $p$-polarized light with overall energy resolution of $125 \mathrm{meV}$.

to maximize the intensity ratio between the bulk and surface states of a single crystal measured in ARPES experiment. Originally it was believed that the IMPF is weakly material dependent, hence the name "universal curve" was coined. Later, Powell et al. ${ }^{43}$ in his review expressed much criticism regarding the "universality," noting that the universal curve should be used, at best, as a semiquantitative guide. This, however, does not discard the claim that at high kinetic energies $E_{\text {kin }}$ the IMPF should smoothly increase as $\sim E_{\text {kin }} / \ln \left(\gamma E_{\text {kin }}\right)$, which can be understood based on the Bethe scattering approach. ${ }^{55,56}$ Therefore to exploit the possibility of varying the bulk sensitivity in our search for the signatures of a CDW in Y-123 spectra we have measured the excitation energy dependence for the $\mathrm{Y}-\Gamma-\mathrm{Y}$ cut, the one where only a parabolic chain band is expected to cross the FL. ${ }^{2}$ Figure 2 contains the intensity distribution for that direction plotted at the FL (a) and $200 \mathrm{meV}$ below the FL (b). While the data in panel (a) exhibit only two parallel features, corresponding to the $\pm k_{\mathrm{F}}$ crossings of the two branches of the parabolic chain band, the intensity distribution in Fig. 2(b) reveals another set of features running parallel with a slightly larger separation. The new features are well visible at low excitation energies $h v \lesssim 920 \mathrm{eV}$, therefore based on the universal dependence of the escape depth one may assume the new feature to be a surface related one. However, the decrease of the photoemission intensity from the surface chain band as compared to the subsurface ones occurs rather abruptly, which is at odds with the universal curve requiring a smooth dependence. Therefore this observation cannot be solely explained in terms of the electron escape depth, and the difference in the $h v$ dependence of the photoemission matrix elements for the the surface and subsurface chains needs to be taken into account. To get an insight into possible reasons for this difference it is enough to consider the simplest estimate
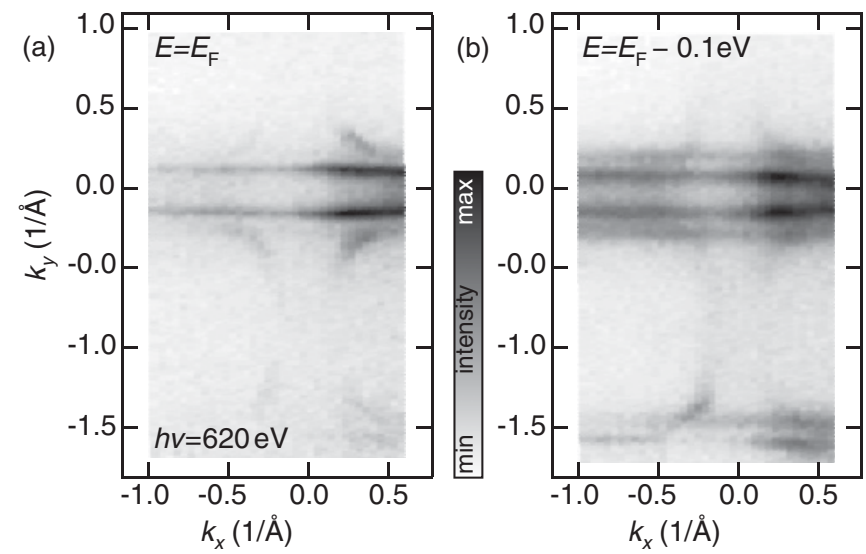

FIG. 3. (a), (b) Momentum intensity distribution at the Fermi level and at $0.1 \mathrm{eV}$ below the $\mathrm{FL}$ for $\mathrm{YBa}_{2} \mathrm{Cu}_{3} \mathrm{O}_{6.6}$. The right image reveals another $1 \mathrm{D}$ band, which contributes practically no spectral weight at the Fermi level. Spectra measured with $p$-polarized light and energy resolution of $90 \mathrm{meV}$.

for the probability of photoemission transition in a form of Fermi's "golden rule,"

$$
w_{i \rightarrow f} \sim \frac{2 \pi}{\hbar}\left|\left\langle f\left|\hat{H}_{\text {pert }}\right| i\right\rangle\right|^{2} \delta\left(E_{f}-E_{i}-\hbar \omega\right),
$$

where the $\hat{H}_{\text {pert }}=-\frac{e i \hbar}{m c}\left(\mathbf{A} \nabla+\frac{1}{2} \operatorname{div} \mathbf{A}\right)$ is the perturbation due to the electromagnetic field. Besides a trivial distinction due to different spatial localization of the surface and subsurface chains there is another factor that introduces a disparity between surface and bulk photoemission. The surface induced spatially oscillating light fields, ${ }^{57-59}$ arising from the mismatch in the dielectric constant between the solid and vacuum, result in a nonzero $\operatorname{div} \mathbf{A}$ term at the surface, consequently modifying the matrix element for the surface localized states. ${ }^{59}$ It was demonstrated experimentally that this term leads to an interference between the bulk and surface emission in $\mathrm{Ag}^{45,60}$ and $\mathrm{Cu}^{61,62}$ and in circular dichroism for $\mathrm{YBa}_{2} \mathrm{Cu}_{2} \mathrm{O}_{7-\delta} .{ }^{31,33}$ In particular, one may speculate that the absence of the new surface chain states in the spectra shown in Fig. 1 would be consistent with the fact that these spectra were measured with $s$-polarized light. For this polarization there is no component of the exciting field $\mathbf{A}(\mathbf{r})$ perpendicular to the sample surface, and hence, owing to the continuity condition for the parallel to the surface component, the surface related term $\operatorname{div} \mathbf{A}$ is negligible, rendering the surface emission effects irrelevant as compared to the case of $p$-polarized light. We want to emphasize that here we have just proposed two possible reasons for the difference in the surface and bulk photoemission matrix elements. Gaining a complete quantitative picture would require a calculation that explicitly accounts for the wave functions and field distribution at the interface, which is beyond the scope of this experimental study.

To establish the dimensionality and topology of this previously overlooked surface state, in Figs. 3(a) and 3(b) we plot a FS measured with $620-\mathrm{eV}$ photons and corresponding isoenergy intensity distribution taken $100 \mathrm{meV}$ below the FL. According to the $h v$ dependence, the new feature is expected to have a significant contribution to the experimental spectra at this energy. As can be seen, the new band practically does not 


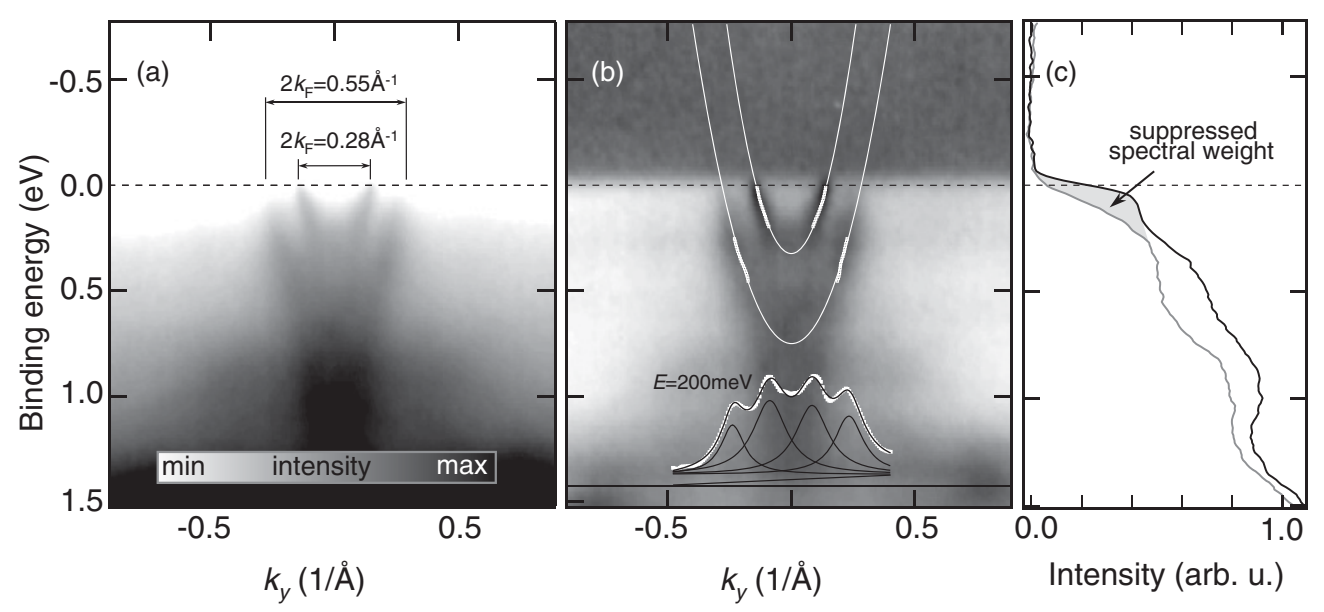

FIG. 4. (a) Energy-momentum cut along the $\mathrm{Y}-\Gamma-\mathrm{Y}$ direction demonstrating two types of chain bands in $\mathrm{YBa}_{2} \mathrm{Cu}_{3} \mathrm{O}_{6.6}$. (b) The same as previous, but each MDC in the image has been normalized to 1 . The small square symbols denote the peak positions obtained in a four-Lorentzian MDC fit. The inset shows typical fit for the MDC at $E_{\mathrm{B}}=200 \mathrm{meV}$. The obtained experimental dispersions are fit to parabolic bands (solid white lines). (c) $k_{\mathrm{F}}$ EDC's for the inner chain band (black line) and outer chain band (gray line). Polarization and resolution are the same as in Fig. 3.

contribute spectral weight at the FL (pseudogapped), though at $100 \mathrm{meV}$ binding energy the band gives rise to two 1D traces similar to those spawned by the chain band. This suggests that the subsurface chains and the pseudogapped surface feature stem from homologous bands with, probably, different spatial localization. Indeed, a detailed analysis of the LDA band structure $^{2}$ shows that there are no other 1D bands except for the chains that the pseudogapped feature could be attributed to. Further, $k_{z}$ dispersion cannot be responsible for the appearance of the second pseudogapped chain band in the spectrum, since along the $\mathrm{Y}-\Gamma-\mathrm{Y}$ direction the calculated $k_{\mathrm{F} \|}$ variation with $k_{z}\left( \pm 4 \% k_{\mathrm{F} \|}\right)$ is notably smaller than the observed splitting.

In Figs. 4(a) and 4(b) we plot the energy-momentum intensity distribution for the $\mathrm{Y}-\Gamma-\mathrm{Y}$ direction. Panel (b) contains the same data set, but each MDC making up the image has been normalized to a fixed value, in order to show the band dispersion in the region that is "overexposed" in panel (a). The inner band with $2 k_{\mathrm{F}} \sim 0.28 \AA^{-1}$ is the chain band that we attribute here to the subsurface chain states, and the outer one, with $2 k_{\mathrm{F}} \sim 0.55 \AA,^{-1}$ is the newly observed band, which we believe to be a surface localized chain band. The suppression of the spectral weight down to about $250 \mathrm{meV}$ for the surface chains as compared to the bulk ones is clearly visible. In panel (c) we additionally compare two energy distribution curves (EDC) taken at the effective $k_{\mathrm{F}}$ of the bulk and surface bands, which once again demonstrate the pseudogaplike suppression of the spectral weight for the surface band. While for the subsurface chains the spectral intensity grows approximately as the Fermi function convoluted with experimental resolution, for the surface band a gradual growth of intensity is observed. The estimated energy scale is about 200-300 meV.

In the case of true CDW gap, using model calculations, it has been demonstrated that new states arise on the order of $E_{\mathrm{F}} \pm \Delta$. For these energies, the CDW component of the local DOS was shown to have a characteristic amplitude and phase. ${ }^{63}$ Extending this result on the pseudogap, we note that the pseudogap nicely agrees with the STM results of Refs. 7 and 12. Namely, the charge corrugations are visible for bias voltages up to $310 \mathrm{meV}$ and practically vanish for bias voltages higher than $480 \mathrm{meV}$. In this regard we can also conclude that another small gap $(\sim 20 \mathrm{meV})$ observed in STM spectra and attributed either to the CDW gap or to the proximity induced superconducting gap is likely to be a superconductivity related one.

It is also informative to see how the observed band dispersion compares to the CDW modulations measured in STM experiment. Simple theory predicts that the CDW wave vector should be twice the Fermi vector, $q_{\mathrm{CDW}}=2 \pi / \lambda_{\mathrm{CDW}}=2 k_{\mathrm{F}}$. To estimate from the photoemission spectrum where the pseudogapped chain band would cross the FL, if there were no CDW instability, we use the result of an MDC fit performed for energies below the gapped region [white symbols in Fig. 4(b)] and extrapolate the experimental points up to the Fermi level assuming a parabolic dispersion. The value thus obtained is $2 k_{\mathrm{F}}=0.55 \AA^{-1}$, which yields $\lambda_{\mathrm{CDW}}=11.4 \AA$. This value nicely compares to the $\lambda=11.2 \AA$, observed in $\mathrm{Zn}$ substituted $\mathrm{Y}-123 .^{12}$ It is also within the range of $\mathrm{CDW}$ periods from 9 to $14 \AA$ reported for a series of $\mathrm{YBa}_{2} \mathrm{Cu}_{3} \mathrm{O}_{7-\delta}$ samples with varying stoichiometry, ${ }^{11}$ approximately corresponding to the sample with $\delta=0.35$ used in that study. In contrast, the $2 k_{\mathrm{F}}=0.28 \AA^{-1}$ wave vector characterizing the subsurface chains would lead to a CDW period of about $22 \AA$, which obviously does not match the STM data. Together with the absence of the (pseudo)gap this once again suggests that CDW modulations, which have been studied in so many details by STM, are due to the $2 k_{\mathrm{F}}=0.55 \AA^{-1}$ instability occurring in the previously overlooked surface chain band. Disorder in the chain structure results in a short-range character of the CDW and the development of the pseudogap in the chain band.

It is remarkable that the chains at the surface develop the CDW state, while the neighboring chains sandwiched between the overdoped and bulk $\mathrm{CuO}_{2}$ layers display no notable signatures of the $\mathrm{CDW}$. One reason for this could 

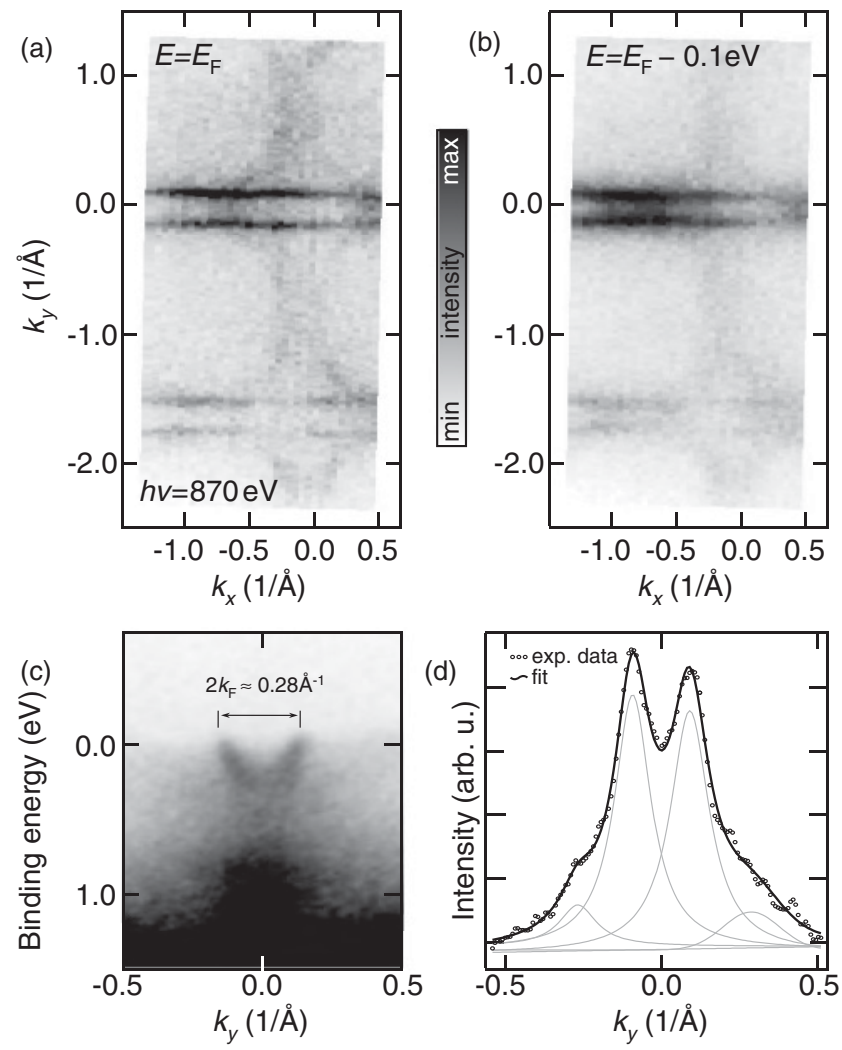

FIG. 5. (a), (b) Momentum intensity distribution at the Fermi level and at $0.1 \mathrm{eV}$ below the FL for $\mathrm{YBa}_{2} \mathrm{Cu}_{3} \mathrm{O}_{6.4}$. (c) Energymomentum cut along the $\mathrm{Y}-\Gamma-\mathrm{Y}$ direction. (d) MDC centered at binding energy of $170 \mathrm{meV}$ and integrated over $\pm 60 \mathrm{meV}$ wide window. In the MDC fit the inner two peaks correspond to the subsurface chains, while the outer two are due to the remnant spectral weight of the heavily disordered surface chains.

be a trivial difference in phonon modes available at the surface and in the bulk. However, commensuration effects appear to be of no less importance. As compared to the incommensurate state, there is an additional energy lowering associated with the commensurability of the CDW state, which is not taken into account in the simplest variant of the CDW theory. ${ }^{6,64}$ The energy gain is given by $E_{\text {comm }}=-\frac{n\left(\epsilon_{\mathrm{F}}\right) \Delta^{2}}{\lambda_{\mathrm{el} \mathrm{ph}}}\left(\frac{\Delta}{D}\right)^{M-2}$. Here $D$ is the bandwidth, $\Delta$ is the CDW gap, $\lambda_{\text {el-ph }}$ is the electronphonon coupling constant, $n\left(\epsilon_{\mathrm{F}}\right)$ is the density of states at the Fermi level, and the commensurability factor is given by $M=\lambda_{\mathrm{CDW}} / a$, where $a$ is the lattice constant. As can be seen, the correction is most significant at low $M$ values. Considering this correction, for the surface chains we have $M \approx 11.4 / 3.89 \approx 2.94$, which is indistinguishable from a commensurate modulation with $M=3$. For the subsurface chains $M=5.77$ is quite large and differs significantly from the nearest commensurate value $M=6$. Consequently the $E_{\text {comm }}$ correction is negligible in this case, which obviously should affect the energy balance, making the CDW phase less favorable for the subsurface chains.

It is well known that the amount of missing oxygen, $\delta$, determines the bulk doping level and consequently the critical temperature of $\mathrm{YBa}_{2} \mathrm{Cu}_{3} \mathrm{O}_{7-\delta}$ samples. ${ }^{65}$ Nonetheless, as shown by ARPES experiments, ${ }^{31,33}$ the hole doping level of $\mathrm{CuO}_{2}$ planes is weakly dependent on $\delta$, being practically fixed at about 0.3 holes per $\mathrm{Cu}$ atom. At the same time oxygen deficiency cannot leave the chain band unaffected. This is well supported by the STM data, ${ }^{11,12}$ demonstrating gradual vanishing of the structure in the chain-terminated surfaces upon increasing oxygen deficiency $\delta$. For these reasons it is also interesting to know how the observed pseudogapped band depends on the chemical composition of $\mathrm{YBa}_{2} \mathrm{Cu}_{3} \mathrm{O}_{7-\delta}$ samples.

Based on the data obtained for $\mathrm{YBa}_{2} \mathrm{Cu}_{3} \mathrm{O}_{6.6}$, we can empirically conclude that the excitation energy in the range between 620 and $920 \mathrm{eV}$ ensures optimal experimental condition for observation of the surface chain band. In Fig. 5 we present spectra measured with $h v=870 \mathrm{eV}$ from the heavily underdoped sample $\mathrm{YBa}_{2} \mathrm{Cu}_{3} \mathrm{O}_{6.4}$. As can be seen, the general appearance of the $\mathrm{CuO}_{2}$ bands and the subsurface chain band is comparable to that observed in $\mathrm{YBa}_{2} \mathrm{Cu}_{3} \mathrm{O}_{6.6}$ [cf. Figs. 3 and 5(a) and 5(b)], even the $2 k_{\mathrm{F}}$ for the subsurface chains amounts to approximately the same value of $0.28 \AA^{-1}$. However, the pseudogapped chain band is practically missing in the spectra. Only a careful analysis of the $Y-\Gamma-Y$ cut [Figs. 5(c) and 5(d)] reveals some remnant spectral weight on both sides of the subsurface chain band. Panel (d) contains an MDC centered at binding energy of $170 \mathrm{meV}$ and integrated over a \pm 60 -meV-wide window. The MDC can be well fit with four Lorentzians, the inner two corresponding to the subsurface chains, and the outer two being due to the remnant spectral weight of the surface chains. We believe that such a vanishing of the surface pseudogaped band in $\mathrm{YBa}_{2} \mathrm{Cu}_{3} \mathrm{O}_{6.4}$ is primarily caused by the substantial oxygen deficiency and hence an enhanced disorder in the cleaved surface chains, which is consistent with the STM data. ${ }^{11,12}$ In other words, in $\mathrm{YBa}_{2} \mathrm{Cu}_{3} \mathrm{O}_{6.4}$ the disorder and amount of defects in the surface chains have reached such a level that at the surface there is no well defined momentum states any more. The effect of disorder is also seen in the subsurface chains, as those are notably broader in momentum as compared to $\mathrm{YBa}_{2} \mathrm{Cu}_{3} \mathrm{O}_{6.6}$, but since the subsurface chains are sandwiched between $\mathrm{CuO}_{2}$ planes the effect of the disorder is not that acute as at the surface.

One may immediately associate the pseudogap with the mysterious pseudogap phase in the phase diagram of the cuprates ${ }^{66,67}$ which alongside the actual suppression of spectral weight is also connected to the anomalous behavior of many physical properties like resistivity, alleged circulating currents, etc. We want to stress that the occurrence of a pseudogap in the spectral function (or density of states) is a far more general phenomenon. Besides cuprates, the pseudogap has been discussed in the context of disordered alloys, ${ }^{68}$ quasicrystals, ${ }^{69-72}$ and charge-density-wave compounds. ${ }^{16,18}$ Apparently the common feature that they all share is the lack of perfect periodicity. This can be either pseudoperiodicity like in the case of quasicrystals, some kind of fluctuation or disorder, which effectively renders a long-range periodic potential into a short-range one. Actually even in cuprates partial suppression of the spectral weight has been connected to short-range antiferromagnetic fluctuations. ${ }^{73}$ Therefore we believe it is an important issue to study how much all the various pseudogaps, currently discussed in literature, have in common. 


\section{CONCLUSIONS}

We have identified the origin of the CDW observed at the surface of Y-123 by STM by detecting a previously overlooked 1D surface band in Y-123, which brings into agreement two complementary methods: ARPES and STM. As a result of a Peierls instability, the surface $\mathrm{CuO}$ chain band develops a short-range CDW state, which results in the appearance of a pseudogap in the one-particle excitation spectrum detected in our ARPES experiment. Both energy and momentum scales measured in STM and ARPES are found to be in agreement with each other.

\section{ACKNOWLEDGMENTS}

We thank F. Dubi and C. Hess for technical support during the measurements. The project was supported in part by DFG Grant No. ZA 654/1-1.
${ }^{1}$ O. K. Andersen, A. I. Liechtenstein, O. Jepsen, and F. Paulsen, Proceedings of the Conference on Spectroscopies in Novel Superconductors, J. Phys. Chem. Solids 56, 1573 (1995).

${ }^{2}$ W. E. Pickett, R. E. Cohen, and H. Krakauer, Phys. Rev. B 42, 8764 (1990).

${ }^{3}$ S. Tomonaga, Prog. Theor. Phys. 5, 544 (1950).

${ }^{4}$ J. M. Luttinger, J. Math. Phys. 4, 1154 (1963).

${ }^{5}$ V. Meden and K. Schönhammer, Phys. Rev. B 46, 15753 (1992).

${ }^{6}$ G. Grüner, Density Waves in Solids (Addison-Wesley, Reading, MA, 1994).

${ }^{7}$ H. L. Edwards, A. L. Barr, J. T. Markert, and A. L. de Lozanne, Phys. Rev. Lett. 73, 1154 (1994).

${ }^{8}$ H. L. Edwards, D. J. Derro, A. L. Barr, J. T. Markert, and A. L. de Lozanne, Phys. Rev. Lett. 75, 1387 (1995).

${ }^{9}$ H. L. Edwards, J. T. Markert, and A. L. de Lozanne, Phys. Rev. Lett. 69, 2967 (1992).

${ }^{10}$ M. Maki, T. Nishizaki, K. Shibata, and N. Kobayashi, J. Phys. Soc. Jpn. 70, 1877 (2001).

${ }^{11}$ M. Maki, T. Nishizaki, K. Shibata, and N. Kobayashi, Phys. Rev. B 65, 140511 (2002).

${ }^{12}$ M. Maki, T. Nishizaki, K. Shibata, and N. Kobayashi, Phys. Rev. B 72, 024536 (2005).

${ }^{13}$ M. Maki, T. Nishizaki, K. Shibata, and N. Kobayashi, Physica C 378-381, 84 (2002).

${ }^{14}$ D. J. Derro, E. W. Hudson, K. M. Lang, S. H. Pan, J. C. Davis, J. T. Markert, and A. L. de Lozanne, Phys. Rev. Lett. 88, 097002 (2002).

${ }^{15}$ G. Urbanik, T. Hänke, C. Hess, B. Büchner, A. Ciszewski, V. Hinkov, C. T. Lin, and B. Keimer, Eur. Phys. J. B 69, 483 (2009).

${ }^{16}$ M. Bovet, D. Popović, F. Clerc, C. Koitzsch, U. Probst, E. Bucher, H. Berger, D. Naumović, and P. Aebi, Phys. Rev. B 69, 125117 (2004).

${ }^{17}$ T. Yokoya, T. Kiss, A. Chainani, S. Shin, and K. Yamaya, Phys. Rev. B 71, 140504 (2005).

${ }^{18}$ S. V. Borisenko, A. A. Kordyuk, A. N. Yaresko, V. B. Zabolotnyy, D. S. Inosov, R. Schuster, B. Büchner, R. Weber, R. Follath, L. Patthey, and H. Berger, Phys. Rev. Lett. 100, 196402 (2008).

${ }^{19}$ S. V. Borisenko, A. A. Kordyuk, V. B. Zabolotnyy, D. S. Inosov, D. Evtushinsky, B. Büchner, A. N. Yaresko, A. Varykhalov, R. Follath, W. Eberhardt, L. Patthey, and H. Berger, Phys. Rev. Lett. 102, 166402 (2009).

${ }^{20}$ J. Schäfer, M. Sing, R. Claessen, E. Rotenberg, X. J. Zhou, R. E. Thorne, and S. D. Kevan, Phys. Rev. Lett. 91, 066401 (2003).

${ }^{21}$ A. Koitzsch, D. S. Inosov, H. Shiozawa, V. B. Zabolotnyy, S. V. Borisenko, A. Varykhalov, C. Hess, M. Knupfer, U. Ammerahl, A. Revcolevschi, and B. Büchner, Phys. Rev. B 81, 113110 (2010).
${ }^{22}$ K. N. Altmann, J. N. Crain, A. Kirakosian, J.-L. Lin, D. Y. Petrovykh, F. J. Himpsel, and R. Losio, Phys. Rev. B 64, 035406 (2001).

${ }^{23}$ L. Bartosch and P. Kopietz, Phys. Rev. Lett. 82, 988 (1999).

${ }^{24}$ L. Bartosch and P. Kopietz, Phys. Rev. B 60, 15488 (1999).

${ }^{25}$ L. Bartosch and P. Kopietz, Phys. Rev. B 62, R16223 (2000).

${ }^{26}$ A. J. Millis and H. Monien, Phys. Rev. B 61, 12496 (2000).

${ }^{27}$ L. Bartosch, Ann. Phys. 10, 799 (2001).

${ }^{28}$ L. Perfetti, H. Berger, A. Reginelli, L. Degiorgi, H. Höchst, J. Voit, G. Margaritondo, and M. Grioni, Phys. Rev. Lett. 87, 216404 (2001).

${ }^{29}$ T. Mizokawa, C. Kim, Z.-X. Shen, A. Ino, A. Fujimori, M. Goto, H. Eisaki, S. Uchida, M. Tagami, K. Yoshida, A. I. Rykov, Y. Siohara, K. Tomimoto, and S. Tajima, Phys. Rev. B 60, 12335 (1999).

${ }^{30}$ T. Mizokawa, K. Nakada, C. Kim, Z.-X. Shen, T. Yoshida, A. Fujimori, S. Horii, Y. Yamada, H. Ikuta, and U. Mizutani, Phys. Rev. B 65, 193101 (2002).

${ }^{31}$ K. Nakayama, T. Sato, K. Terashima, H. Matsui, T. Takahashi, M. Kubota, K. Ono, T. Nishizaki, Y. Takahashi, and N. Kobayashi, Phys. Rev. B 75, 014513 (2007).

${ }^{32}$ V. B. Zabolotnyy, S. V. Borisenko, A. A. Kordyuk, J. Geck, D. S. Inosov, A. Koitzsch, J. Fink, M. Knupfer, B. Büchner, S.-L. Drechsler, H. Berger, A. Erb, M. Lambacher, L. Patthey, V. Hinkov, and B. Keimer, Phys. Rev. B 76, 064519 (2007).

${ }^{33}$ V. B. Zabolotnyy, S. V. Borisenko, A. A. Kordyuk, D. S. Inosov, A. Koitzsch, J. Geck, J. Fink, M. Knupfer, B. Büchner, S.-L. Drechsler, V. Hinkov, B. Keimer, and L. Patthey, Phys. Rev. B 76, 024502 (2007).

${ }^{34}$ M. A. Hossain, J. D. F. Mottershead, D. Fournier, A. Bostwick, J. L. McChesney, J. L. McChesney, E. Rotenberg, R. Liang, W. N. Hardy, G. A. Sawatzky, I. S. Elfimov, D. A. Bonn, and A. Damascelli, Nat. Phys. 4, 527 (2008).

${ }^{35}$ V. N. Strocov, T. Schmitt, U. Flechsig, T. Schmidt, A. Imhof, Q. Chen, J. Raabe, R. Betemps, D. Zimoch, J. Krempasky, X. Wang, M. Grioni, A. Piazzalunga, and L. Patthey, J. Synchrotron Radiat. 17, 631 (2010).

${ }^{36}$ V. N. Strocov, M. Kobayashi, M. Shi, X. Wang, T. Schmitt, and L. Patthey (unpublished).

${ }^{37}$ V. Hinkov, S. Pailhes, P. Bourges, Y. Sidis, A. Ivanov, A. Kulakov, D. P. Lin, C. T. Chen, C. Bernhard, and B. Keimer, Nature (London) 430, 650 (2004).

${ }^{38}$ R. Hesper, L. H. Tjeng, A. Heeres, and G. A. Sawatzky, Phys. Rev. B 62, 16046 (2000).

${ }^{39}$ K. Pasanai and W. A. Atkinson, Phys. Rev. B 81, 134501 (2010).

${ }^{40}$ D. H. Lu, D. L. Feng, N. P. Armitage, K. M. Shen, A. Damascelli, C. Kim, F. Ronning, Z.-X. Shen, D. A. Bonn, R. Liang, W. N. Hardy, A. I. Rykov, and S. Tajima, Phys. Rev. Lett. 86, 4370 (2001). 
${ }^{41}$ A. Damascelli, D. H. Lu, K. M. Shen, N. P. Armitage, F. Ronning, D. L. Feng, C. Kim, Z.-X. Shen, T. Kimura, Y. Tokura, Z. Q. Mao, and Y. Maeno, Phys. Rev. Lett. 85, 5194 (2000).

${ }^{42}$ M. P. Seach and W. A. Dench, Surf. Interface Anal. 1, 2 (1979).

${ }^{43}$ C. J. Powell, A. Jablonski, I. S. Tilinin, S. Tanuma, and D. R. Penn, J. Electron Spectrosc. Relat. Phenom. 98-99, 1 (1999).

${ }^{44}$ I. S. Tilinin, Phys. Rev. B 53, 547 (1996).

${ }^{45}$ E. D. Hansen, T. Miller, and T.-C. Chiang, Phys. Rev. B 55, 1871 (1997).

${ }^{46}$ E. E. Krasovskii, W. Schattke, P. Jiříček, M. Vondráček, O. V. Krasovska, V. N. Antonov, A. P. Shpak, and I. Bartoš, Phys. Rev. B 78, 165406 (2008).

${ }^{47}$ N. Barrett, E. E. Krasovskii, J.-M. Themlin, and V. N. Strocov, Phys. Rev. B 71, 035427 (2005).

${ }^{48}$ D. Fournier, G. Levy, Y. Pennec, J. L. McChesney, A. Bostwick, E. Rotenberg, R. Liang, W. N. Hardy, D. A. Bonn, I. S. Elfimov, and A. Damascelli, Nat. Phys. 6, 905 (2010).

${ }^{49}$ Y. Sassa, M. Radović, M. Månsson, E. Razzoli, X. Y. Cui, S. Pailhès, S. Guerrero, M. Shi, P. R. Willmott, F. Miletto Granozio, J. Mesot, M. R. Norman, and L. Patthey, Phys. Rev. B 83, 140511 (2011).

${ }^{50}$ V. Zabolotnyy, S. Borisenko, A. Kordyuk, J. Geck, D. Inosov, A. Koitzsch, J. Fink, M. Knupfer, and B. B. Büchner, Physica C 460-462, 888 (2007).

${ }^{51}$ D. Katagiri, K. Seki, R. Eder, and Y. Ohta, Phys. Rev. B 83, 165124 (2011).

${ }^{52}$ S. Basak, T. Das, H. Lin, J. Nieminen, M. Lindroos, R. S. Markiewicz, and A. Bansil, Phys. Rev. B 80, 214520 (2009).

${ }^{53}$ D. S. Inosov, J. Fink, A. A. Kordyuk, S. V. Borisenko, V. B. Zabolotnyy, R. Schuster, M. Knupfer, B. Büchner, R. Follath, H. A. Dürr, W. Eberhardt, V. Hinkov, B. Keimer, and H. Berger, Phys. Rev. Lett. 99, 237002 (2007).

${ }^{54}$ D. S. Inosov, R. Schuster, A. A. Kordyuk, J. Fink, S. V. Borisenko, V. B. Zabolotnyy, D. V. Evtushinsky, M. Knupfer, B. Büchner, R. Follath, and H. Berger, Phys. Rev. B 77, 212504 (2008).
${ }^{55}$ S. Tanuma, C. J. Powell, and D. R. Penn, Surf. Interface Anal. 37, 1 (2005).

${ }^{56}$ H. Bethe, Ann. Phys. 397, 325 (1930).

${ }^{57}$ H. J. Levinson and E. W. Plummer, Phys. Rev. B 24, 628 (1981).

${ }^{58}$ B. C. Meyers and T. E. Feuchtwang, Phys. Rev. B 27, 2030 (1983).

${ }^{59}$ B. Feuerbacher and R. F. Willis, J. Phys. C 9, 169 (1976).

${ }^{60}$ T. Miller, W. E. McMahon, and T.-C. Chiang, Phys. Rev. Lett. 77, 1167 (1996).

${ }^{61}$ T. Michalke, A. Gerlach, K. Berge, R. Matzdorf, and A. Goldmann, Phys. Rev. B 62, 10544 (2000).

${ }^{62}$ F. Pforte, T. Michalke, A. Gerlach, A. Goldmann, and R. Matzdorf, Phys. Rev. B 63, 115405 (2001).

${ }^{63}$ W. Sacks, D. Roditchev, and J. Klein, Appl. Phys. A 66, S925 (1998).

${ }^{64}$ P. A. Lee, T. M. Rice, and P. W. Anderson, Solid State Commun. 14, 703 (1974).

${ }^{65}$ R. Liu, B. W. Veal, A. P. Paulikas, J. W. Downey, H. Shi, C. G. Olson, C. Gu, A. J. Arko, and J. J. Joyce, Phys. Rev. B 45, 5614 (1992).

${ }^{66}$ N. P. Armitage, P. Fournier, and R. L. Greene, Rev. Mod. Phys. 82, 2421 (2010).

${ }^{67}$ P. A. Lee, N. Nagaosa, and X.-G. Wen, Rev. Mod. Phys. 78, 17 (2006).

${ }^{68}$ T. C. McGill and J. Klima, Phys. Rev. B 5, 1517 (1972).

${ }^{69}$ J. C. Phillips, Phys. Rev. B 47, 2522 (1993).

${ }^{70}$ T. Fujiwara and T. Yokokawa, Phys. Rev. Lett. 66, 333 (1991).

${ }^{71}$ X. Wu, S. W. Kycia, C. G. Olson, P. J. Benning, A. I. Goldman, and D. W. Lynch, Phys. Rev. Lett. 75, 4540 (1995).

${ }^{72}$ J. Hafner and M. Krajčí, Phys. Rev. B 47, 11795 (1993).

${ }^{73}$ I. A. Nekrasov, N. S. Pavlov, E. Z. Kuchinskii, M. V. Sadovskii, Z. V. Pchelkina, V. B. Zabolotnyy, J. Geck, B. Büchner, S. V. Borisenko, D. S. Inosov, A. A. Kordyuk, M. Lambacher, and A. Erb, Phys. Rev. B 80, 140510 (2009). 